\title{
Triple functionalisation of single-walled carbon nanotubes with doxorubicin, a monoclonal antibody, and a fluorescent marker for targeted cancer therapy
}

\author{
Elena Heister ${ }^{1 *}$, Vera Neves ${ }^{1}$, Carmen Tîlmaciu ${ }^{2}$, Kamil Lipert $^{3}$, Vanesa Sanz Beltrán ${ }^{1}$, \\ Helen M. Coley ${ }^{1}$, S. Ravi P. Silva ${ }^{4}$, and Johnjoe McFadden ${ }^{1}$
}

\footnotetext{
${ }^{1}$ Faculty of Health and Medical Sciences, University of Surrey, Guildford, GU27XH, UK ${ }^{2}$ CIRIMAT-LCMIE, CNRS-UMR 5085, Université Paul Sabatier, 118 Route de Narbonne, 31077 Toulouse, Cedex 9, France

${ }^{3}$ Leibniz Institute for Solid State and Materials Research (IFW), Helmholtzstraße 20, 01069 Dresden, Germany

${ }^{4}$ Nanoelectronics Centre, Advanced Technology Institute, University of Surrey, Guildford, GU27XH, UK
}

\begin{abstract}
Single-walled carbon nanotubes (SWCNTs) have been identified as a transporter for anticancer drugs, as they are capable of penetrating mammalian cell membranes and allow for a high drug loading due to their nanoscale dimensions and high aspect ratio. In addition, they can assist the targeting of therapeutic agents to the desired site of action by conjugation to antibodies or ligands of cancer cell surface receptors, which increases the effectiveness of the treatment and reduces side effects. In this work, we present a method for the triple
\end{abstract}


functionalisation of oxidised SWCNTs with the anti-cancer drug doxorubicin, a monoclonal antibody, and a fluorescent marker at non-competing binding sites. The proposed methodology allows for the targeted delivery of the anticancer drug to cancer cells and the visualisation of the cellular uptake of SWCNTs by confocal microscopy. We show that the complex is efficiently taken up by cancer cells with subsequent intracellular release of doxorubicin, which then translocates to the nucleus while the nanotubes remain in the cytoplasm.

\section{Introduction}

The development of novel anti-cancer therapies is often limited by administration problems of drugs, such as insolubility, inefficient distribution, lack of selectivity, and the inability of drugs to cross cellular barriers. Currently, most of these problems are subject of intense studies and in this context, many different types of drug delivery systems have been investigated, including quantum dots, silica nanoparticles, dendrimers, micelles, molecular conjugates, and liposomes [1-4]. Within the family of nanomaterials, carbon nanotubes have emerged as a novel tool for the delivery of therapeutic molecules into cells [5]. Carbon nanotubes are undoubtedly one of the most striking discoveries in the quest for new materials in recent years, as these unique structures possess tremendous strength [6], an extreme aspect ratio, and are excellent thermal and electrical conductors [7,8]. Apart from that, carbon nanotubes can immobilise therapeutic molecules, such as proteins, antibodies, DNA or drugs on their surface $[9,10]$ or in the hollow cavity $[11]$ and are capable of penetrating mammalian cell membranes [12], which makes them ideal candidates for drug delivery systems. 
In this article, we present a new approach to functionalise single-walled carbon nanotubes (SWCNTs) with three different agents for multimodal drug delivery. This includes the anticancer drug doxorubicin, a monoclonal antibody for molecular targeting, and the fluorescent marker fluorescein, all of which are attached to oxidised SWCNTs at non-competing binding sites. Doxorubicin was chosen as a therapeutic agent, as it is a widely used anti-cancer drug for the treatment of many cancers and furthermore possesses a fluorescent hydroxysubstituted anthraquinone chromophore with an emission spectrum ranging from 500 to 750 $\mathrm{nm}$ that enables intracellular tracking of the drug by confocal microscopy. The monoclonal antibody recognizes carcinoembryonic antigen (CEA), which is a tumour marker for the identification of metastatic disease following surgical resection and is relevant for a variety of adenocarcinomas, such as colon cancer. Fluorescein is used to label SWCNTs in order to colocalise them inside cells by confocal microscopy next to doxorubicin.

The attachment of the three agents was accomplished by two different chemical approaches; non-covalent and covalent binding. Non-covalent chemistry has the advantage that it preserves the structure of SWCNTs and thus their unique properties. However, a noncovalent bond is susceptible to environmental factors, such as $\mathrm{pH}$ and salt concentration, and is in general less stable than a covalent bond. In contrast to this, covalent attachment of molecules to carbon nanotubes depends on the introduction of chemically reactive groups to their relatively inert $\mathrm{sp}^{2}$ structure, which can only be achieved by harsh treatments, such as oxidation with concentrated inorganic acids [13], fluorination by elemental fluorine [14] or a 1,3 dipolar cycloaddition reaction [15] and inevitably introduces defects to the nanotube structure. 
On the basis of these considerations, non-covalent functionalisation was chosen for attachment of doxorubicin to allow for its release after cellular uptake, whereas covalent functionalisation was employed to attach fluorescein and CEA antibodies to oxidised SWCNTs. The latter was achieved by using the hydrophilic protein bovine serum albumin (BSA) as a multifunctional linker. In total, BSA possesses 60 amino groups in lysine chains and 99 carboxylic groups as part of glutamic and aspartic acid residues. Those residues located on the protein surface are available for conjugation reactions and thus provide two different types of binding sites for the covalent attachment of molecules. In our approach, BSA is conjugated to carboxylic groups of oxidised SWCNTs by one of its amine groups, to NHS-fluorescein via the remaining amine groups, and to monoclonal CEA antibodies via its carboxylic groups. Since multiple copies of fluorescein and antibodies can be attached to one BSA molecule, the degree of coupling is increased manifold. Figure 1 shows a schematic illustration of the structure, which we propose for the nanotube-drug-BSA conjugates. The distribution of functional groups after acid oxidation of carbon nanotubes has already been investigated in previous studies, which showed that functional groups are found at the ends of the tubes and along the sidewalls $[16,17]$. Results obtained by atomic force microscopy (AFM) demonstrate a similar distribution for BSA molecules attached to functional groups of oxidised, doxorubicin-loaded SWCNTs in our experiments (insert of Figure 1). 

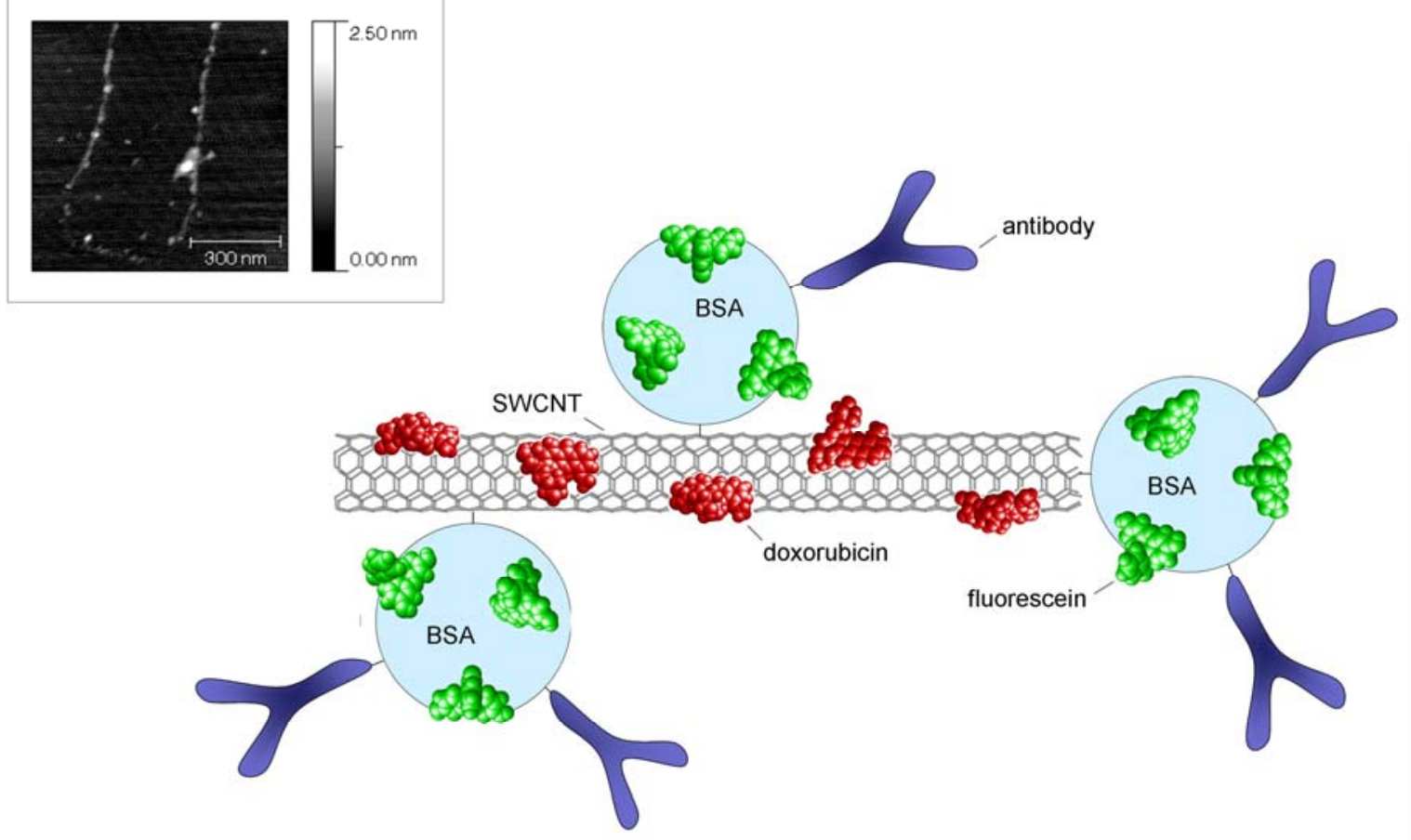

Figure 1 Schematic illustration of the doxorubicin-fluorescein-BSA-antibody-SWCNT complexes (red=doxorubicin, green=fluorescein, light blue=BSA, dark blue=antibodies). Insert: AFM image of doxorubicin-fluorescein-BSA-SWCNT complexes (without antibodies).

The cellular uptake of drug-antibody-SWCNT complexes was studied by means of confocal microscopy using CEA-expressing WiDr colon cancer cells. The design of the nanotube-drug complexes is based on the hypothesis that functionalised SWCNTs are taken up by WiDr cells by endocytosis. Following uptake, the lower $\mathrm{pH}$ inside endosomes is expected to trigger the release of doxorubicin from the nanotube due to increased hydrophilicity of the drug, which can then translocate to the nucleus and exert its cytotoxic action.

\section{Experimental Details}




\subsection{Material}

SWCNTs were purchased from NanoLab, Inc. (produced by CVD using iron (ferrocene) as catalyst, purity $>60 \%$, length 1-5 $\mu$ m, Lot No. 90907). WiDr human colon cancer cells were obtained from LGC Promochem (Teddington, UK; Catalog No. CCL-218). The monoclonal CEA antibody with specificity for CD66e was purchased from AbD Serotec (MCA1744F). MEM (31095-029) and Opti-MEM medium (31985-047) were obtained from Invitrogen (Paisley, UK). Doxorubicin hydrochloride (44583), 5(6)-carboxyfluorescein Nhydroxysuccinimide ester (NHS-fluorescein, 21878), bovine serum albumin (BSA, A3294), 1-ethyl-3-[3-dimethyl-aminopropyl] carbodiimide hydrochloride (EDC, E1769), Nhydroxysulfo-succinimide (sulfo-NHS, 56485), 2-mercaptoethanol (63700), and ethanolamine hydrochloride (E6133) were purchased from Sigma-Aldrich (Poole, UK).

\subsection{Oxidation and Purification of SWCNTs}

As-prepared SWCNTs are usually contaminated with metal catalyst particles, amorphous carbon and graphitic nanoparticles. However, the application of carbon nanotubes for biomedical purposes has created a demand for high purity material, especially if the sidewalls of carbon nanotubes are to serve as platform for the non-covalent attachment of biomolecules. Li et al. have compared different oxidation methods for CVD-synthesized SWCNTs and found that nitric acid pre-sonication, followed by refluxing in a mixture of concentrated acids yield the best results with respect to purity [18]. This two-step process was slightly adapted for the work presented here. In the first step, $100 \mathrm{mg}$ of SWCNTs were incubated in $20 \mathrm{~mL}$ of concentrated nitric acid and sonicated with a tip sonicator for $6 \mathrm{x} 10 \mathrm{~s}$ 
to disentangle the tubes and break up bundles. Subsequently, the mixture was incubated in a $95^{\circ} \mathrm{C}$ water bath for $2 \mathrm{~h}$. To remove dissolved metal catalyst particles, the oxidation treatment was followed by a centrifugation step at $3000 \mathrm{~g}$ for $30 \mathrm{~min}$. After discarding the supernatant, the purified tubes were washed twice with bi-distilled water. In the second step, the purified sample of SWCNTs ( 100 mg) was dispersed in a 3:1 mixture of concentrated nitric and sulfuric acid and sonicated for 6x 10s with a tip sonicator. Subsequently, the mixture was refluxed at $110{ }^{\circ} \mathrm{C}$ for $2 \mathrm{~h}$, followed by three washing steps with water and centrifugation at $3000 \mathrm{~g}$ for $30 \mathrm{~min}$ to remove excess acid in the supernatant. Finally, the oxidised SWCNTs were vacuum filtered using a $0.2 \mu \mathrm{m}$ polycarbonate filter (Whatman) until the eluate was clear and of neutral $\mathrm{pH}$. The filter cake was dried overnight at room temperature, weighed, and resuspended in water to a concentration of $5 \mathrm{mg} / \mathrm{mL}$. This material will henceforth be referred to as "oxSWCNTs".

\subsection{Characterisation of pristine and oxidised SWCNTs}

The term "carbon nanotube” does not describe a simple, chemical structure, but a whole class of materials, which vary in their numbers of walls, diameter distribution, length distribution, chirality, purity, catalyst material, impurity species and defects. Thus, characterisation of the applied material is crucial in order to ensure quality and reproducibility. The nanotubes in this work were characterised by scanning electron microscopy (SEM), high resolution transmission electron microscopy (HR-TEM), atomic force microscopy (AFM), Raman spectroscopy, and thermogravimetric analyis (TGA).

\subsubsection{SEM}


All SEM images in this study were obtained using a FEI 200 Nova NanoSEM system. Powder samples were prepared by placing a small amount of SWCNT powder onto a piece of double-sided adhesive tape stuck to a metal stub. Liquid samples were prepared by placing a $2 \mu \mathrm{L}$ drop of a $10 \mu \mathrm{g} / \mathrm{mL}$ SWCNT suspension onto a small piece of silicon stuck to a metal stub and evaporating residual water using a hot plate. The metal stubs holding the samples were then placed into the vacuum chamber of the microscope and images acquired using a voltage of $10 \mathrm{kV}$ and a spot size of 3.0 to 5.0 .

\subsection{2. $H R-T E M$}

The HR-TEM work presented in this report was carried out using a High Resolution Transmission Electron Microscope JEOL-EM 2100. For sample preparation, oxSWCNTs were diluted with ethanol and briefly sonicated in a water bath for dispersion. 3-5 drops of this suspension were then placed onto a 300 mesh copper TEM grid and excess liquid was removed by touching one edge of the grid with filter paper.

\subsection{3. $A F M$}

For AFM sample preparation, oxSWCNTs were diluted with water to obtain a concentration of $10 \mu \mathrm{g} / \mathrm{mL}$. A droplet of $2 \mu \mathrm{L}$ was placed onto a freshly cleaved mica substrate $\left(1 \mathrm{~cm}^{2}\right)$ and was dried with help of compressed air. AFM measurements were performed using a Veeco Dimension 3100 Atomic Force Microscope in tapping mode. To obtain a length distribution, several AFM images were taken at a frame size of $5 \mu \mathrm{m} \times 5 \mu \mathrm{m}$ and the lengths of 500 nanotubes was measured using “Gwyddion 2.9”, a free SPM data visualization and imaging tool released under the GNU General Public License. 


\subsubsection{Raman Spectroscopy}

Samples for Raman spectroscopy were prepared by placing a small quantity of carbon nanotube powder or a droplet of an oxSWCNT suspension between a glass slide and a cover slip. Measurements were performed using a NT-MDT NTEGRA Spectra Probe NanoLaboratory with excitation at $633 \mathrm{~nm}$.

\subsection{5. $T G A$}

For a typical TGA experiment, $1 \mathrm{mg}$ of nanotube material was placed in the sample holder in the furnace of a Rheometric Scientific TG 760 series and the material was heated up at a rate of $10^{\circ} \mathrm{C} / \mathrm{min}$, while the weight was measured and recorded continuously.

\subsection{Triple functionalisation of SWCNTs with doxorubicin, fluorescein, and CEA antibodies}

\section{Labeling of BSA with fluorescein}

$3 \mathrm{mg}$ NHS-fluorescein (10 mg/mL in DMSO) was mixed with $150 \mathrm{mg}$ BSA in sodium phosphate buffer $20 \mathrm{mM} \mathrm{pH} \mathrm{8.5,} \mathrm{followed} \mathrm{by} \mathrm{incubation} \mathrm{for} 2 \mathrm{~h}$ in darkness at room temperature while stirring. To remove excess fluorescein, the mixture was filtered and washed repeatedly using Amicon Ultra ${ }^{\circledR} 30 \mathrm{kDa}$ centrifugal filtration devices (Millipore) until the eluate was clear. The fluorescein-BSA conjugates were resuspended in sodium phosphate buffer $20 \mathrm{mM}(\mathrm{pH} 7.4)$ at a BSA concentration of $5 \mathrm{mg} / \mathrm{mL}$. 
The degree of labeling (fluorescein/BSA molar ratio) was determined by separately calculating the protein and fluorophore molar concentrations of the conjugate based on absorbance measurements and expressing these concentrations as a ratio. Absorbance at 280 $\mathrm{nm}\left(A_{280}\right)$ was used to determine the protein concentration in the sample. However, because fluorescent dyes also absorb at $280 \mathrm{~nm}$, a correction factor must be used to adjust for the amount of $A_{280}$ contributed by the dye. The correction factor $(C F)$ equals the $A_{280}$ of the dye divided by the $A_{\max }$ of the dye and was determined to be 0.26 for NHS-fluorescein (data not shown).

In the first step, the molar concentration of BSA $\left(c_{B S A}\right)$ was calculated according to equation (1) which is derived from Beer-Lambert's Law $(A=\varepsilon \cdot c \cdot l$ ) with $A$ being the absorbance of the respective molecules, $C F$ being the correction factor, $\varepsilon$ being the molar extinction coefficient, $l$ being the path length of the cuvette and $D F$ being the dilution factor:

$$
c_{B S A}=\frac{A_{B S A, 280}-\left(A_{\text {fluorescein }, 494} \cdot C F\right)}{\varepsilon_{B S A} \cdot l} \cdot D F
$$

In the second step, the degree of labeling (moles fluorescein per mole protein) was calculated according to equation (2):

$$
\frac{n_{\text {fluorescein }}}{n_{B S A}}=\frac{A_{\text {fluorescein }, 494}}{\varepsilon_{\text {fluorescein }} \cdot c_{B S A}} \cdot D F
$$


Non-covalent attachment of doxorubicin to oxidised SWCNTS

$150 \mu \mathrm{L}$ of a $5 \mathrm{mg} / \mathrm{mL}$ oxSWCNT suspension was dispersed in $15 \mathrm{~mL}$ sodium phosphate buffer $20 \mathrm{mM}$ pH 8.5 and $3 \mathrm{~mL}$ of a $5 \mathrm{mM}$ doxorubicin hydrochloride solution was added. The mixture was sonicated in a water bath for 15 min and incubated overnight while stirring. Unbound doxorubicin was removed by filtering and washing using Amicon Ultra ${ }^{\circledR} 30 \mathrm{kDa}$ centrifugal filter devices (Millipore). Finally, the doxorubicin-loaded SWCNTs were resuspended in $15 \mathrm{~mL}$ sodium phosphate buffer $20 \mathrm{mM} \mathrm{pH} 7.4$ at a SWCNT concentration of $50 \mu \mathrm{g} / \mathrm{mL}$ and $5 \mathrm{~mL}$ of these complexes were kept as a control sample, whereas the other 10 $\mathrm{mL}$ were used for further conjugation steps. In order to determine how much doxorubicin was bound to the nanotubes, the eluate of the centrifugation steps containing unbound doxorubicin were collected and analysed by UV/vis absorption spectroscopy by measuring the absorbance at the first excitation maximum of doxorubicin at $490 \mathrm{~nm}$.

\section{Connection of fluorescein-labeled BSA and doxorubicin-loaded SWCNTs}

$10 \mathrm{~mL}$ doxorubicin-loaded SWCNTs were dispersed in $10 \mathrm{~mL}$ sodium phosphate buffer 20 $\mathrm{mM} \mathrm{pH}$ 8.5. If necessary, the $\mathrm{pH}$ was adjusted to 8.5 by means of a trisodium phosphate 20 $\mathrm{mM}$ solution. Next, $1 \mathrm{~mL}$ of a $20 \mathrm{mM}$ EDC solution and $1 \mathrm{~mL}$ of a $50 \mathrm{mM}$ sulfo-NHS solution were added and the mixture was incubated for $5 \mathrm{~min}$ at room temperature. To quench excess EDC, $70 \mu \mathrm{L}$ 2-mercaptoethanol was added to a final concentration of $20 \mathrm{mM}$. To start the coupling reaction, $10 \mathrm{~mL}$ of the fluorescein-labeled BSA solution $(5 \mathrm{mg} / \mathrm{mL}$ BSA) was added and the reaction allowed to proceed for $2 \mathrm{~h}$ at room temperature. Finally, 1 $\mathrm{mL}$ of a $250 \mathrm{mM}$ ethanolamine hydrochloride solution was added to quench the reaction. The nanotube complexes were washed by vacuum filtration using $0.2 \mu \mathrm{M}$ polycarbonate filters 
(Whatman) and were resuspended in $10 \mathrm{~mL}$ sodium phosphate buffer $20 \mathrm{mM}$ pH 7.4. $5 \mathrm{~mL}$ of these complexes were kept for experiments and the remaining $5 \mathrm{~mL}$ were used for the last conjugation step.

Attachment of monoclonal CEA antibodies to doxorubicin-fluorescein-BSA-SWCNT conjugate

$5 \mathrm{~mL}$ of the doxorubicin-fluorescein-BSA-SWCNT complexes in water were mixed with 5 mL sodium phosphate buffer $50 \mathrm{mM} \mathrm{pH}$ 8.5. If necessary, the $\mathrm{pH}$ was adjusted to 8.5 by means of a trisodium phosphate $20 \mathrm{mM}$ solution. Next, $500 \mu \mathrm{L}$ of a $20 \mathrm{mM}$ EDC solution and $500 \mu \mathrm{L}$ of a $50 \mathrm{mM}$ sulfo-NHS solution were added and the mixture was incubated for 5 min at room temperature. To quench excess EDC, $35 \mu \mathrm{L}$ 2-mercaptoethanol was added to a final concentration of $20 \mathrm{mM}$. Subsequently, $5 \mathrm{~mL}$ of a $1 \mu \mathrm{g} / \mathrm{mL}$ anti-CEA antibody solution was added and the reaction allowed to proceed for $2 \mathrm{~h}$ at room temperature in darkness while stirring. Finally, $500 \mu \mathrm{L}$ of a $250 \mathrm{mM}$ ethanolamine hydrochloride solution was added to quench the reaction and the nanotube complexes were washed by vacuum filtration using 0.2 $\mu \mathrm{M}$ polycarbonate filters (Whatman) and resuspended in $5 \mathrm{~mL}$ sodium phosphate buffer 20 $\mathrm{mM} \mathrm{pH}$ 7.4.

\subsection{Delivery of the drug-SWCNT conjugates to WiDr colon cancer cells}

The WiDr human colon cancer cell line was derived from a 78-year old female Caucasian and expresses carcinoembryonic antigen (CEA) at a rate of $118 \mathrm{ng} / 10^{6}$ cells/10 days according to LGC Promochem's product information sheet. Growth medium is Eagle's Minimal Essential Medium (MEM) containing $2 \mathrm{mM}$ L-glutamine, which was modified to 
contain $1.0 \mathrm{mM}$ sodium pyruvate, $0.1 \mathrm{mM}$ non-essential amino acids, $10 \%$ fetal bovine serum, and penicillin/streptomycin (100 units/mL penicillin and $100 \mu \mathrm{g} / \mathrm{mL}$ streptomycin in the final formulation). The medium is formulated for use with a $5 \% \mathrm{CO}_{2}$ in air atmosphere. For the cell studies, WiDr cells were grown on cover slips in a 6-well plate until $70-80 \%$ confluent. The prepared doxorubicin-fluorescein-BSA-SWCNT conjugates and the controls were mixed with Opti-MEM medium at a 1:1 ratio (500 $\mu \mathrm{L}$ complexes $+500 \mu \mathrm{L}$ medium), resulting in final SWCNT concentrations of $25 \mu \mathrm{g} / \mathrm{mL}$. The mixtures were sonicated for $3 \mathrm{x}$ 10s using a tip sonicator and remaining agglomerates removed by centrifugation for $60 \mathrm{~s}$ at 3000 g. Next, WiDr cells were incubated with $1 \mathrm{~mL}$ of the sample or control solutions in Opti-MEM medium for $4 \mathrm{~h}$ at $37^{\circ} \mathrm{C}$. The cells were then washed three times with PBS and fixed by incubation with freshly prepared $4 \%$ paraformaldehyde solution at room temperature for $30 \mathrm{~min}$. Finally, the cells were washed twice with PBS and mounted on a glass slide.

Confocal microscopy was carried out using a Zeiss LSM 510 confocal microscope equipped with an Argon/2 laser in multi-channel mode. The excitation wavelength was set to $488 \mathrm{~nm}$ for fluorescein and doxorubicin. Emission of fluorescein was detected between 500 and 530 $\mathrm{nm}$ and emission of doxorubicin was detected from 650 to $710 \mathrm{~nm}$. Images were taken at $63 \mathrm{x}$ magnification by means of a C-Apochromat 63x/1.4 Oil objective.

\section{Results and Discussion}

\subsection{Characterisation of oxidised SWCNTs}


Pristine and oxidised Nanolab SWCNTs were characterised by SEM, HR-TEM, AFM, Raman spectroscopy, and TGA in order to evaluate their purity and their physical and chemical properties.
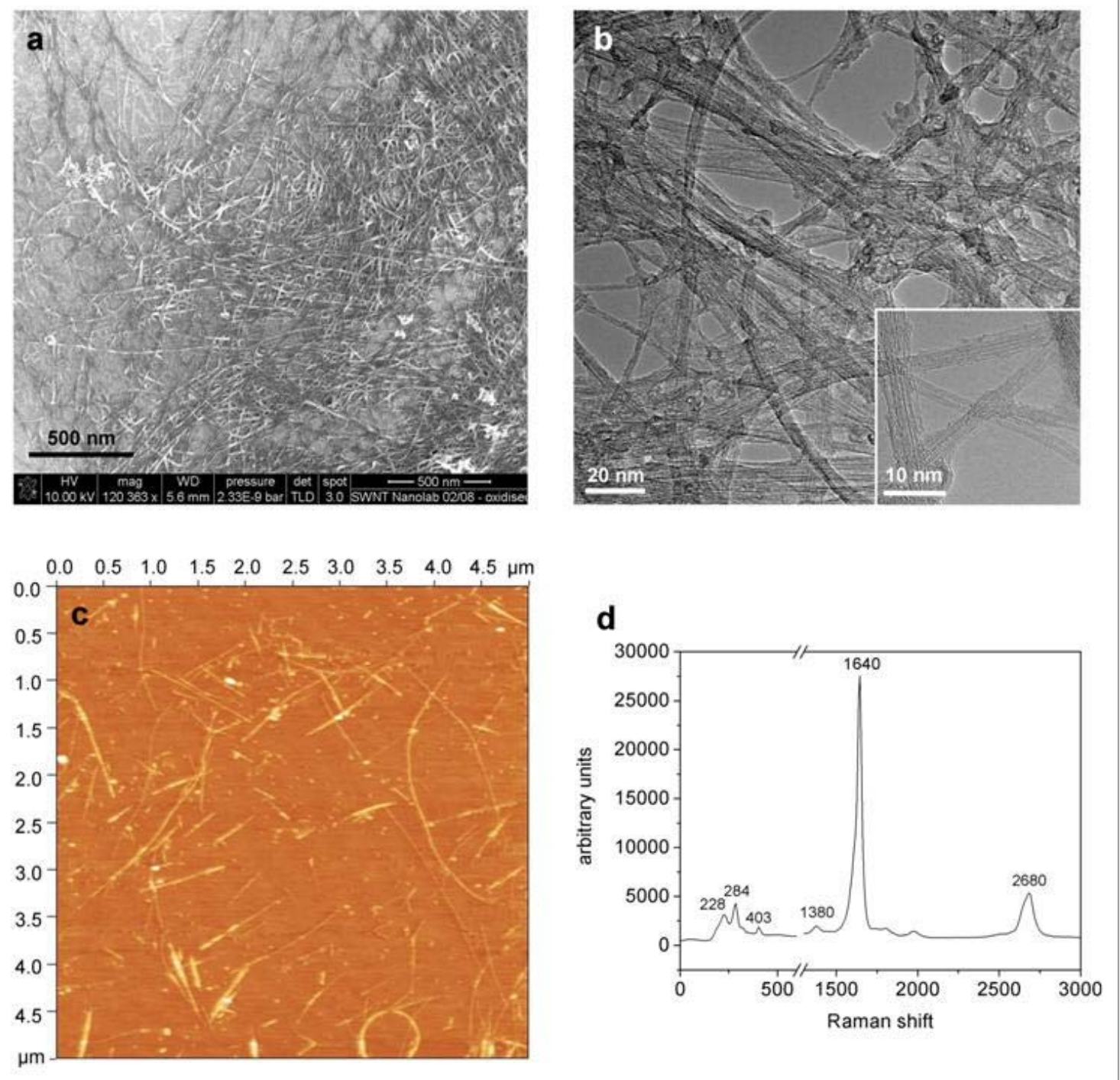

Figure 2 Characterisation of oxidised SWCNTs: a) SEM image, magnification $=120 \mathrm{~K}$; b) HR-TEM image at two magnifications; c) AFM image (frame size $5 \mathrm{\mu m}^{2}$ ); d) Raman spectrum $($ excitation $=633 \mathrm{~nm})$ 
The SEM image of oxidised SWCNTs (Fig. 2a) demonstrates that acid oxidation entangles clumps of nanotubes that are present in the bulk sample. The oxidised SWCNTs seem to form small bundles which are covered with a thin layer of a material that has been shown to consist of decomposed products of carbonaceous impurities [19], such as partially oxidised graphitic fragments, and amorphous carbon. Thermal oxidation can be applied to remove these remaining impurities; however, they improve the solubilisation properties of carbon nanotubes by acting as a surfactant [20]. The TEM picture in Fig. $2 \mathrm{~b}$ reveals more detailed information about the bundling state of the nanotubes. Apart from single nanotubes, bundles of up to 15 nanotubes can be observed, which are again covered with the same oxidation debris. AFM analysis of carbon nanotubes was applied to extract a height profile and to generate a length distribution of the oxidised SWCNTs (Fig. 2c, Fig. 3). The height profile features seven SWCNT bundles with diameters in the range of 2.0 to $4.0 \mathrm{~nm}$. Given that the average diameter of a single SWCNT is about $1 \mathrm{~nm}$, this corresponds to bundles consisting of 3 to 10 nanotubes. The length distribution was used to quantify the extent of shortening by the oxidation process. The maximum of the size distribution after oxidation lies at $450 \mathrm{~nm}$ and $83 \%$ of the tubes were found to be between $200 \mathrm{~nm}$ and $1000 \mathrm{~nm}$ long. Considering that the lengths of SWCNTs in the pristine sample ranged from 1 to $5 \mu \mathrm{m}$, this corresponds to a shortening of $80 \%$. The Raman spectrum (Fig. 2d) shows all features that are characteristic for SWCNTs: the radial breathing mode (RBM), the D-band and the G-band [21,22]. The RBM results from low-energy radial vibrations of carbon atoms in the nanotube backbone and hence its frequency is inversely proportional to the tube diameter according to $\omega=223.5 / d+12.5$, where $d$ is the tube diameter in nm and $\omega$ is Raman shift in wave numbers [23]. The RBM in the Raman spectrum of Fig. 2d ranges from $170 \mathrm{~cm}^{-1}$ to $410 \mathrm{~cm}^{-1}$; 
corresponding to a diameter distribution between 0.56 and $1.42 \mathrm{~nm}$ with an average diameter of approximately $1 \mathrm{~nm}$. Maximum intensities are observed at $228 \mathrm{~cm}^{-1}, 284 \mathrm{~cm}-1$, and 403 $\mathrm{cm}-1$; relating to accumulated diameters at $1.06 \mathrm{~nm}, 0.82 \mathrm{~nm}$, and $0.57 \mathrm{~nm}$. The disorderinduced D-band at $1380 \mathrm{~cm}^{-1}$ indicates the presence of defective, $\mathrm{sp}^{3}$ hybridised sites on SWCNTs and the G-band at $1640 \mathrm{~cm}^{-1}$ is a tangential vibrational mode characteristic to all graphitic materials. The G/D intensity ratio is a good indicator for the quality of bulk samples. Here, the G/D ratio was found to be 13.95, demonstrating a low quantity of defects.
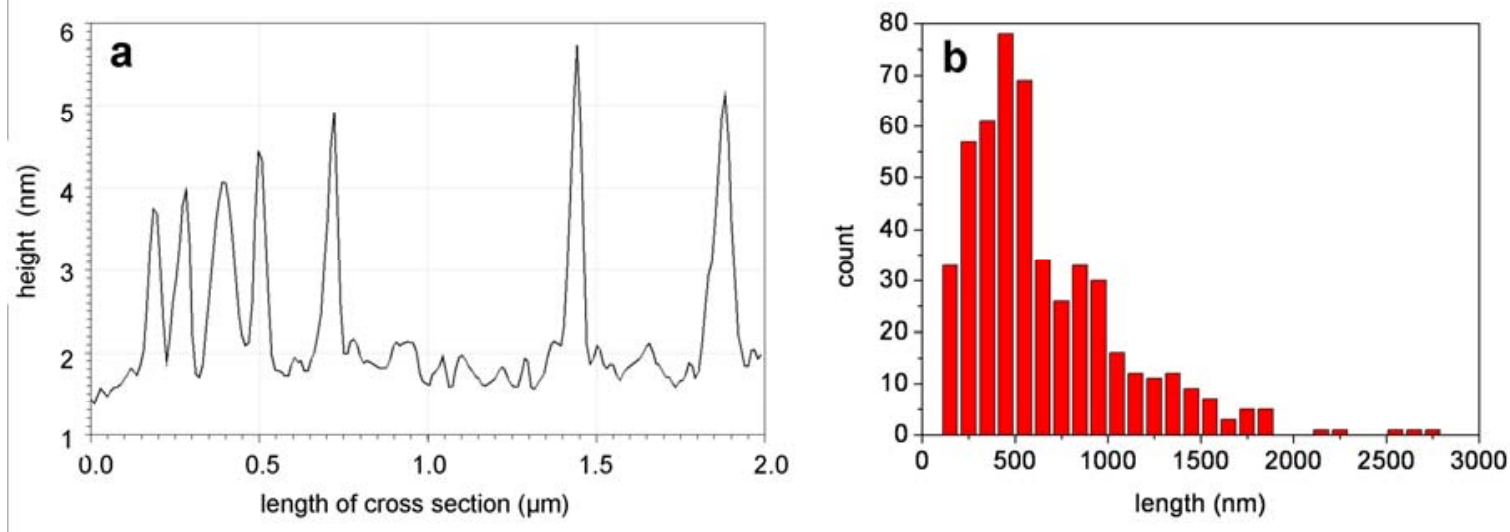

Figure 3 Height profile (a) of oxSWCNTs extracted from Fig. 2c and length distribution (b) of oxSWCNTs $(n=500)$

Last, but not least, oxidised SWCNTs were analysed by TGA to determine the amount of metal catalyst in the bulk sample. The metal residue was found to be $11.2 \%$ for pristine SWCNTs and $11.6 \%$ for oxidised SWCNTs (data not shown). This indicates that the present metallic catalyst particles are encapsulated in carbon shells (so-called nanoonions) [24], which protect them from acid oxidation, but also prevent them from being released in biological environments and causing toxic effects. 


\subsection{Triple functionalisation of SWCNTs with doxorubicin, fluorescein, and CEA antibodies}

We developed a method for the triple functionalisation of SWCNTs, which allows for the attachment of a monoclonal antibody for targeting purposes, an anti-cancer drug, and a fluorescent dye at non-competing binding sites to enable visualisation of cellular uptake. The functionalisation procedure is a critical process comprising 4 steps and can easily result in many kinds of unwanted crosslinking reactions. Hence, the reaction parameters have to be selected and adjusted carefully. In the first step, the protein BSA is labeled with the aminereactive dye NHS-fluorescein. Here, it is crucial to find the optimal NHS-fluorescein concentration in order to leave enough free amino groups on the BSA for the later attachment of carbon nanotubes. However, leaving too many free binding sites would lead to crosslinking of nanotubes via BSA and thus to precipitation. Optimisation of the fluorescein/BSA ratio was performed by testing three different weight ratios (1:20, 1:50, and 1:100) in their binding properties to SWCNTs (see next paragraph). Furthermore, the degree of fluorescein labeling was determined by UV/vis absorption spectroscopy (see equations 1 and 2). A 1:20 weight ratio of fluorescein/BSA resulted in a degree of labeling of 3.99, whereas a 1:50 weight ratio correlated with a degree of 2.19 and a 1:100 weight ratio with a degree of 1.19 .

In the second step of the coupling reaction, the anti-cancer drug doxorubicin is noncovalently attached to the sidewalls of oxidised carbon nanotubes via $\pi$-stacking and hydrophilic interactions with carboxylic groups. The strength of this non-covalent binding is 
$\mathrm{pH}$ dependent: At a low $\mathrm{pH}$, the amino group in the sugar moiety of doxorubicin is protonated, which increases the molecule's hydrophilicity and thus its solubility in water. However, at a higher $\mathrm{pH}$, the amino group becomes deprotonated, resulting in stronger hydrophobic interactions with the nanotubes' side walls and lower solubility in water. Liu et al. have attached doxorubicin non-covalently to the side walls of SWCNTs by overnight incubation at pH 9 [25]. However, Janssen et al. reported that doxorubicin hydrochloride has its maximum stability at $\mathrm{pH} 4$ [26] and processing the drug at pH 9 may cause its degradation and thus the loss of its therapeutic efficiency. Thus, to ensure a high drug loading whilst maintaining doxorubicin's therapeutic properties, the reaction was carried out at a slightly lower $\mathrm{pH}$ of 8.5. According to Beijnen et al., who generated a $\mathrm{pH}$-dependent decomposition profile for doxorubicin at $50{ }^{\circ} \mathrm{C}$ corrected for buffer and ionic strength influences [27], the decomposition rate of doxorubicin at pH 8.5 is approximately 35\% less than at $\mathrm{pH} 9.0$.

The amount of doxorubicin bound to the nanotubes was calculated by determining the quantity of unbound doxorubicin in the eluate of the filtration step by UV/vis absorption spectroscopy. It was found that $87.5 \%$ of doxorubicin had attached to the nanotubes, whereas $12.5 \%$ had been washed off. In terms of weight, the ratio of doxorubicin to oxidised SWCNTs is 20:1, which demonstrates the enormous binding capacity of the nanotubes. Liu et al. have determined a weight ratio of $4: 1$ in a similar experiment [25] - however, the surface of their (non-oxidised) SWCNTs was already covered with phospholipid-PEGmolecules to a certain extent, which were previously applied to solubilise the nanotubes.

In the third step, the fluorescein-labeled BSA and doxorubicin-loaded SWCNTs are conjugated. In order to avoid non-specific binding of BSA to the nanotubes' sidewalls, it is 
crucial to load them with doxorubicin before conjugation with fluorescein-BSA. In order to find out the optimal fluorescein/BSA ratio, three fluorescein/BSA complexes at different weight ratios (1:20, 1:50, and 1:100) were tested with respect to their binding to SWCNTs in a qualitative and quantitative way. The corresponding molar ratios are 7.0, 2.8, and 1.4. We expected a high fluorescein/BSA ratio to result in low binding due to most of the BSA's binding sites being occupied by fluorescein molecules. Accordingly, the optimal ratio would correlate to exactly one free binding site per BSA molecule, whereas several free binding sites would lead to crosslinking of nanotubes, resulting in agglomeration and a lower percentage of binding. These assumptions could be confirmed experimentally with optimal results having been achieved for a 1:50 ratio, which was thus used for all further experiments.

Table 1 Influence of the fluorescein/BSA ratio on the binding of the respective conjugates to SWCNTs

\begin{tabular}{lccc}
\hline Fluorescein/BSA ratio (weight) & $\mathbf{1 : 2 0}$ & $\mathbf{1 : 5 0}$ & $\mathbf{1 : 1 0 0}$ \\
\hline $\begin{array}{l}\text { Degree of labeling } \\
\text { \% of fluorescein-BSA bound to }\end{array}$ & 3.85 & 2.30 & 1.26 \\
$\begin{array}{l}\text { SWCNTs at the described } \\
\text { experimental conditions }\end{array}$ & $19.33 \%$ & $54.81 \%$ & $14.56 \%$ \\
Agglomeration & little & little & much \\
\hline
\end{tabular}

In the last step, monoclonal antibodies are attached to the still unoccupied carboxylic groups of the BSA (now part of the doxorubicin-fluorescein-BSA-SWCNT complexes). Here, it has to be considered that whenever two protein ligands undergo a coupling reaction catalysed by EDC and sulfo-NHS, excessive EDC has to be quenched by 2-mercaptoethanol, as otherwise 
carboxylic groups of both coupling partners (BSA and CEA antibodies) would be activated and cause extensive crosslinking.

\subsection{Delivery of the drug-SWCNT conjugates to WiDr colon cancer cells}

To study the uptake of doxorubicin-fluorescein-BSA-SWCNT complexes by WiDr colon cancer cells and investigate the suitability of functionalised SWCNTs as a drug delivery system, WiDr cells were incubated with the nanotube-drug complexes for $4 \mathrm{~h}$. Afterwards, laser scanning confocal microscopy was applied to track the location of doxorubicin and the fluorescein-labeled SWCNTs inside cells. Confocal microscopy is a special type of fluorescence microscopy, which only detects light within the focal plane by eliminating outof-focus information via point illumination and a pinhole in front of the detector.
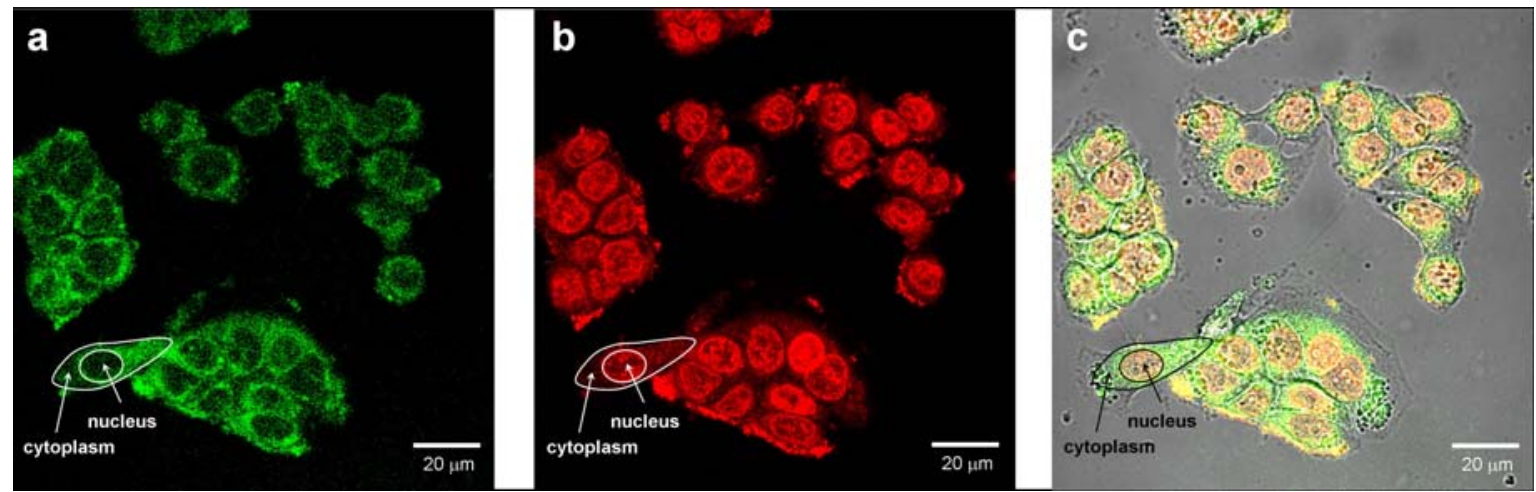

Figure 4 Confocal image of WiDr cells incubated with doxorubicin-fluorescein-BSASWCNT complexes (a=emission measured at 500-530 nm (fluorescein), b=emission measured at 650-710 $\mathrm{nm}$ (doxorubicin), c=transmitted light image showing all channels)

As mentioned earlier, the whole drug delivery experiment is based on the hypothesis that functionalised SWCNTs are taken up by WiDr cells by endocytosis. We expected the lower 
$\mathrm{pH}$ inside endosomes to trigger the release of doxorubicin from the nanotubes due to increased hydrophilicity. Indeed, Fig. 4 clearly demonstrates, that doxorubicin (red) does not colocalise any longer with the fluorescein-labeled SWCNTs (green) after internalization by WiDr cells, but was found to accumulate in the nuclei of cells (seen as round structures positioned in the in the centre of the cell). The fluorescently-labeled nanotubes, however, are mainly observed outside the nuclei within the cytoplasm. Areas in the cell where doxorubicin and fluorescein-labeled nanotubes colocalise can be observed along the border between the cytoplasm and the nucleus, the so-called "nuclear envelope", and on the cell membrane. The delivery efficiency in this experiment was $100 \%$, meaning that all cells have taken up the SWCNT complexes.

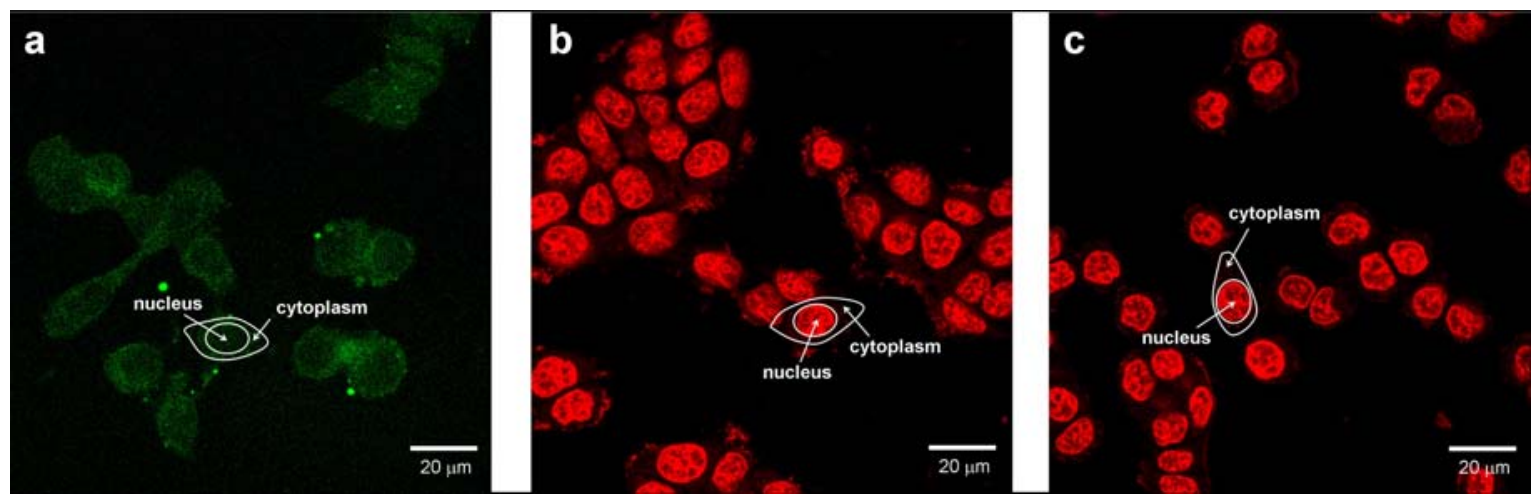

Figure 5 Confocal images of controls: a) fluorescein-BSA; b) doxorubicin-SWCNTs; c) doxorubicin $10 \mu \mathrm{M}$

Several controls were performed to corroborate these findings, including fluorescein-BSA conjugates, doxorubicin-SWCNT complexes without fluorescein-BSA, and doxorubicin alone. Fig. 5a shows a low level of fluorescence, thus indicating that fluorescein-BSA conjugates are only able to enter cells to a very small extent, which demonstrates that 
SWCNTs play an important role as a delivery system. However, when comparing Fig. 5b and 5c it becomes obvious that this is not the case for doxorubicin: due to its molecular structure, the drug is able to cross cell membranes without the help of a carrier system and accumulates in the cell nuclei, as shown before. Nevertheless, the nanotubes still play an important role, as they enable molecular targeting via the attachment of monoclonal antibodies and are crucial for the delivery of drugs that are not taken up by cells under normal conditions.

Apart from this, more work needs to be done with regard to the question whether carbon nanotubes are able to enter nuclei of cells. This study clearly showed that the nanotubes remained in the cytoplasm under the applied conditions. The same behavior was demonstrated by Kam et al., who studied the uptake of Cy3-DNA-wrapped SWCNTs by Hela cells and detected fluorescence in the cytoplasm only [28]. However, Pantarotto et al. have conducted a study where two different carbon nanotube conjugates were introduced into human and murine fibroblasts and found that water-soluble SWCNTs conjugated to fluorescein isothiocyanate (FITC) accumulated in the cytoplasm, whereas SWCNTs conjugated to a FITC-labeled peptide were observed to distribute inside the nucleus [12]. Furthermore, Cheng et al. recently showed that FITC-PEG-SWCNTs accumulated in the nuclei of several mammalian cell lines [29]. This indicates that the ability of CNTs to cross cellular barriers, such as the nuclear envelope, is dependent on the type of functionalisation.

\section{Conclusion}

This work presents a novel functionalisation approach to equip oxidised SWCNTs with three different agents for multimodal drug delivery. It is demonstrated that SWCNTs can 
successfully transport a potent anti-cancer drug to human cancer cells with subsequent translocation of the drug to the nucleus, while the SWCNTs remain in the cytoplasm. This finding suggests that SWCNTs may be used to enhance cellular pharmacokinetics and have considerable implications for improving anti-cancer drug delivery. However, targeting and toxicity of SWCNTs remain important issues that need to be examined in greater depth and will be addressed in future studies. The triple functionalisation methodology presented in this article already incorporates the attachment of monoclonal antibodies for molecular targeting and current work is in progress in order to investigate, whether the uptake of CEA antibodytagged SWCNT conjugates by WiDr colon cancer cells is increased in comparison to untagged conjugates.

\section{Acknowledgements}

This work has been performed in the framework of the FP6 Marie Curie Research Training Network CARBIO (Multifunctional carbon nanotubes for biomedical applications) funded by the European Union. 


\section{References}

[1] Panyam J, Labhasetwar V. Biodegradable nanoparticles for drug and gene delivery to cells and tissue. Advanced Drug Delivery Reviews. 2003 Feb 24;55(3):329-47.

[2] Okuda T, Kawakami S, Akimoto N, Niidome T, Yamashita F, Hashida M. PEGylated lysine dendrimers for tumor-selective targeting after intravenous injection in tumorbearing mice. Journal of Controlled Release. 2006 Dec 1;116(3):330-6.

[3] Merdan T, Kopecek J, Kissel T. Prospects for cationic polymers in gene and oligonucleotide therapy against cancer. Advanced Drug Delivery Reviews. 2002 Sep 13;54(5):715-58.

[4] Park JW. Liposome-based drug delivery in breast cancer treatment. Breast Cancer Research. 2002;4(3):93-7.

[5] Klingeler R, Hampel S, Büchner B. Carbon nanotube based biomedical agents for heating, temperature sensoring and drug delivery. International Journal of Hyperthermia. 2008;24(6):496-505.

[6] Yakobson BI, Avouris P. Mechanical properties of carbon nanotubes. In: Dresselhaus MS, Dresselhaus G, Avouris P, eds. Carbon nanotubes: Synthesis, structure, properties, and applications. Berlin/Heidelberg: Springer 2001:287-327.

[7] Odom TW, Huang JL, Kim P, Lieber CM. Structure and electronic properties of carbon nanotubes. Journal of Physical Chemistry B. 2000 Apr 6;104(13):2794-809.

[8] Hone J, Llaguno MC, Biercuk MJ, Johnson AT, Batlogg B, Benes Z, et al. Thermal properties of carbon nanotubes and nanotube-based materials. Applied Physics A: Materials Science \& Processing. 2002 Mar;74(3):339-43.

[9] Lin Y, Allard LF, Sun YP. Protein-affinity of single-walled carbon nanotubes in water. Journal of Physical Chemistry B. 2004 Mar 25;108(12):3760-4.

[10] Shim M, Kam NWS, Chen RJ, Li YM, Dai HJ. Functionalization of carbon nanotubes for biocompatibility and biomolecular recognition. Nano Letters. 2002 Apr;2(4):2858. 
[11] Hampel S, Kunze D, Haase D, Kramer K, Rauschenbach M, Ritschel M, et al. Carbon nanotubes filled with a chemotherapeutic agent: a nanocarrier mediates inhibition of tumor cell growth. Nanomedicine. 2008 Apr;3(2):175-82.

[12] Pantarotto D, Briand JP, Prato M, Bianco A. Translocation of bioactive peptides across cell membranes by carbon nanotubes. Chemical Communications. 2004 Jan $7(1): 16-7$.

[13] Liu J, Rinzler AG, Dai HJ, Hafner JH, Bradley RK, Boul PJ, et al. Fullerene pipes. Science. 1998 May 22;280(5367):1253-6.

[14] Mickelson ET, Huffman CB, Rinzler AG, Smalley RE, Hauge RH, Margrave JL. Fluorination of single-wall carbon nanotubes. Chemical Physics Letters. 1998 Oct 30;296(1-2):188-94.

[15] Georgakilas V, Tagmatarchis N, Pantarotto D, Bianco A, Briand JP, Prato M. Amino acid functionalisation of water soluble carbon nanotubes. Chemical Communications. 2002(24):3050-1.

[16] Azamian BR, Coleman KS, Davis JJ, Hanson N, Green MLH. Directly observed covalent coupling of quantum dots to single-wall carbon nanotubes. Chemical Communications. 2002(4):366-7.

[17] Cech J, Curran SA, Zhang DH, Dewald JL, Avadhanula A, Kandadai M, et al. Functionalization of multi-walled carbon nanotubes: Direct proof of sidewall thiolation. Physica Status Solidi B: Basic Solid State Physics. 2006 Nov;243(13):3221-5.

[18] Li Y, Zhang XB, Luo JH, Huang WZ, Cheng JP, Luo ZQ, et al. Purification of CVD synthesized single-wall carbon nanotubes by different acid oxidation treatments. Nanotechnology. 2004 Nov;15(11):1645-9.

[19] Nagasawa S, Yudasaka M, Hirahara K, Ichihashi T, Iijima S. Effect of oxidation on single-wall carbon nanotubes. Chemical Physics Letters. 2000 Oct 6;328(4-6):374-80.

[20] Rosca ID, Watari F, Uo M, Akaska T. Oxidation of multiwalled carbon nanotubes by nitric acid. Carbon. 2005 Dec;43(15):3124-31. 
[21] Saito R, Kataura H. Optical properties and Raman spectroscopy of carbon nanotubes. In: Dresselhaus MS, Dresselhaus G, Avouris P, eds. Carbon nanotubes: Synthesis, structure, properties, and applications. Berlin/Heidelberg: Springer 2001:213-46.

[22] Dresselhaus MS, Dresselhaus G, Saito R, Jorio A. Raman spectroscopy of carbon nanotubes. Physics Reports: Review Section of Physics Letters. 2005 Mar;409(2):4799.

[23] Bachilo SM, Strano MS, Kittrell C, Hauge RH, Smalley RE, Weisman RB. Structureassigned optical spectra of single-walled carbon nanotubes. Science. 2002 Dec 20;298(5602):2361-6.

[24] Pumera M. Carbon nanotubes contain residual metal catalyst nanoparticles even after washing with nitric acid at elevated temperature because these metal nanoparticles are sheathed by several graphene sheets. Langmuir. 2007 May 22;23(11):6453-8.

[25] Liu Z, Sun XM, Nakayama-Ratchford N, Dai HJ. Supramolecular chemistry on water-soluble carbon nanotubes for drug loading and delivery. ACS Nano. 2007 Aug;1(1):50-6.

[26] Janssen MJH, Crommelin DJA, Storm G, Hulshoff A. Doxorubicin decomposition on storage - Effect of $\mathrm{pH}$, type of buffer and liposome encapsulation. International Journal of Pharmaceutics. 1985;23(1):1-11.

[27] Beijnen JH, Vanderhouwen OAGJ, Underberg WJM. Aspects of the degradation kinetics of doxorubicin in aqueous solution. International Journal of Pharmaceutics. 1986 Oct;32(2-3):123-31.

[28] Kam NWS, O'Connell M, Wisdom JA, Dai HJ. Carbon nanotubes as multifunctional biological transporters and near-infrared agents for selective cancer cell destruction. Proceedings of the National Academy of Sciences of the United States of America. 2005 Aug 16;102(33):11600-5.

[29] Cheng J, Fernando KAS, Veca LM, Sun Y-P, Lamond AI, Lam YW, et al. Reversible accumulation of PEGylated single-walled carbon nanotubes in the mammalian nucleus. ACS Nano. 2008;2(10):2085-94. 


\section{List of Figures}

Figure 1 Schematic illustration of the doxorubicin-fluorescein-BSA-antibody-SWCNT complexes (red=doxorubicin, green=fluorescein, light blue=BSA, dark blue=antibodies). Insert: AFM image of doxorubicin-fluorescein-BSA-SWCNT complexes (without antibodies) ........................................................................... 5

Figure 2 Characterisation of oxidised SWCNTs: a) SEM image, magnification = 120K; b) HR-TEM image at two magnifications; c) AFM image (frame size $5 \mu m^{2}$ ); d) Raman spectrum $($ excitation $=633 \mathrm{~nm})$

Figure 3 Height profile (a) of oxSWCNTs extracted from Fig. 2c and length distribution (b) of oxSWCNTs $(\mathrm{n}=500)$

Figure 4 Confocal image of WiDr cells incubated with doxorubicin-fluorescein-BSASWCNT complexes ( $\mathrm{a}=$ emission measured at 500-530 $\mathrm{nm}$ (fluorescein), $\mathrm{b}=$ emission measured at 650-710 nm (doxorubicin), c=transmitted light image showing all channels)

Figure 5 Confocal images of controls: a) fluorescein-BSA; b) doxorubicin-SWCNTs; c) doxorubicin $10 \mu \mathrm{M}$.

\section{List of Tables}

Table 1 Influence of the fluorescein/BSA ratio on the binding of the respective conjugates to SWCNTs 